\title{
Avaliação dos resultados do exame papanicolaou não procurados por mulheres de uma Unidade Básica de Saúde no município de Cajazeiras - Paraíba
}

\author{
Lyvia Maria Fernandes $^{1}$ (D), Francisco Assis Cavalcante Junior ${ }^{2}$ (D) Rogéria
} Mônica Seixas Xavier de Abreu $^{3}$ (D), Marcos Alan Sousa Barbosa ${ }^{1}$ (D) \& Marilena Maria de Souza ${ }^{4}$ (I)

(1) Universidade Federal de Campina Grande, Centro de Formação de Professores, Unidade Acadêmica de Ciências da Vida, Rua Sérgio Moreira de Figueiredo, Casas Populares 58900-000, Cajazeiras, Paraíba, Brasil. E-mail: lyvia_fernandes@outlook.com

(2) Universidade Federal de Campina Grande, Centro de Formação de Professores, Unidade Acadêmica de Enfermagem, Rua Sérgio Moreira de Figueiredo, Casas Populares 58900-000, Cajazeiras, Paraíba, Brasil. E-mail: junior_cz228@hotmail.com

(3) Universidade Regional do Cariri, Avenida Dário Rabêlo, Santo Antônio 63500-000, Iguatu, Ceará, Brasil. E-mail: seixasxavier@hotmail.com

(4) Universidade Federal de Campina Grande, Centro de Formação de Professores, Escola Técnica de Saúde de Cajazeiras, Rua Sérgio Moreira de Figueiredo, Casas Populares 58900-000, Cajazeiras, Paraíba, Brasil. E-mail: marilenacarolino@gmail.com

Fernandes L.M., Cavalcante Junior F.A., Abreu R.M.S.X., Barbosa M.A.S. \& Souza M.M. (2021) Avaliação dos resultados do exame papanicolaou não procurados por mulheres de uma Unidade Básica de Saúde no município de Cajazeiras - Paraíba. Pesquisa e Ensino em Ciências Exatas e da Natureza, 5(edição especial 1): e1648. http://dx.doi.org/10.29215/pecen.v5i1.1648

Responsabilidade editorial: Cícero Emanoel Alves Leite. Recebido: 04 dezembro 2020. Aceito: 04 janeiro 2021. Publicado: 11 fevereiro 2021

Resumo: Este trabalho é uma pesquisa documental, exploratória, com abordagem quantitativa e teve como objetivo evidenciar as infecções sexualmente transmissíveis a partir dos resultados do exame Papanicolau não procurados por mulheres em uma Unidade Básica de Saúde, no município de Cajazeiras - Paraíba, no período de janeiro de 2010 a dezembro de 2015. A amostra foi composta por 113 resultados de exames Papanicolau, entre eles 30 (26\%) tiveram resultado positivo para Infecções Sexualmente Transmissíveis (ISTs), sendo que um deles apresentou positividade para duas ISTs. O resultado negativo para a doença foi encontrado em $82(73 \%)$ dos laudos, ressaltando que houve mais 1 (1\%) resultado de alteração celular possivelmente não neoplásica. Dentre os resultados positivos para ISTs, $22(71 \%)$ corresponderam aos casos de Vaginose e 9 (29\%) aos casos de Candidíase. Denota-se que existe um número acentuado de mulheres que não retornaram à Unidade Básica de Saúde estudada para recolher os laudos do exame Papanicolau que apresentaram ISTs. Evidencia-se a relevância e necessidade do retorno das pacientes para recolherem o resultado do exame e a apresentação do mesmo ao profissional competente que, em casos positivos, poderá iniciar o tratamento precocemente, impedindo a transmissão e evitando outras infecções e prováveis complicações.

Palavras-chave: Papanicolau, Infecções Sexualmente Transmissíveis, rastreamento.

Assessment of paper examination results not looked for by women from a Basic Health Unit in the municipality of Cajazeiras - Paraíba

Abstract: Documentary, exploratory research, with a quantitative approach, aimed to show how sexually transmitted infections from the Pap smear results not sought by women from a Basic Health Unit, in the municipality of Cajazeiras-PB from January 2010 to December 2015. The sample consisted of 113 Pap smear results, among which $30(26 \%)$ had positive results for Sexually Transmitted Infections 
(STIs), one of which was positive for two STIs. The negative results for these diseases were found in 82 $(73 \%)$ of the reports, emphasizing that there was an additional $1(1 \%)$ result of a cellular change, possibly non-neoplastic. Of those who tested positive for STIs, $22(71 \%)$ corresponded to cases of Vaginosis and 9 $(29 \%)$ to cases of Candidiasis. It is noted that there is an accentuated number of women who did not return to the Basic Health Unit studied to collect the Pap smear reports that have STIs. The relevance and necessity of the patients' return are evident to collect the results of the examination and the presentation of the same to the competent professional who, in positive cases, may start the treatment early, preventing transmission and avoiding other infections and probable complications.

Keywords: Pap smear, Sexually Transmitted Infections, mass screening.

A Atenção Primária à Saúde é reconhecida como o eixo estruturante do Sistema Único de Saúde, sendo enfatizada, cada vez mais, sua função de congregar um conjunto de ações de promoção e proteção à saúde, prevenção de agravos, diagnóstico, tratamento, reabilitação e manutenção da saúde nas dimensões coletiva e individual. Entre as ações desenvolvidas pelas equipes de Atenção Básica, destacam-se as ações relacionadas ao controle dos cânceres do colo de útero (BRASIL 2013).

O câncer do colo do útero é o segundo tipo de neoplasia mais incidente nas Regiões Norte (21.20/100 mil), Nordeste (17.62/100 mil) e Centro-Oeste (15.92/100 mil) do Brasil, sem considerar os tumores de pele não melanoma (BRASIL 2020).

O seu rastreamento se baseia na história natural da doença e no reconhecimento de que o câncer evolui a partir de lesões precursoras (lesões intraepiteliais escamosas de alto grau e adenocarcinoma in situ), que podem ser detectadas e tratadas adequadamente, impedindo a progressão para neoplasia. No Brasil, o método principal e mais amplamente utilizado para esse rastreamento é o teste de Papanicolaou (exame citopatológico do colo do útero) (BRASIL 2016).

O Papanicolaou também pode trazer resultados positivos caso a paciente apresente certos tipos de infecção sexualmente transmissível (IST). As ISTs são constantes, possuem diversas causas, sinais, sintomas e exercem influência na qualidade de vida dos portadores, nas interações pessoais, familiares e sociais. Algumas ISTs possuem elevadas taxas de incidência e prevalência, exibem maiores complicações em mulheres e favorecem a infecção pelo vírus da imunodeficiência humana (HIV). As ISTs estão usualmente associadas à culpa, estigma, discriminação e violência, por causas biológicas, psicológicas, sociais e culturais (BRASIL 2015).

A rotina recomendada para o rastreamento é a repetição do exame Papanicolaou a cada três anos, após dois exames normais consecutivos realizados com um intervalo de um ano, com início da coleta aos 25 anos de idade para as mulheres que já tiveram ou têm atividade sexual. Esses exames devem seguir até os 64 anos e, naquelas mulheres sem história prévia de doença neoplásica pré-invasiva, interrompidos quando tiverem pelo menos dois exames negativos consecutivos nos últimos cinco anos (BRASIL 2016).

Ademais, ainda há um número expressivo de mulheres que não retornam para receber o resultado do exame. Entre os motivos encontrados em estudos, há fatores relacionados à mulher, ao profissional que realiza o atendimento e ao serviço (Greenwood et al. 2006). Tendo em vista esse contexto, surgiu a seguinte pergunta norteadora para a pesquisa: Qual é o número de exames que não foram retirados na Unidades de Básica de Saúde e quais infecções estão presentes nestes laudos? Respondendo a essa questão, evidencia-se a relevância do retorno das pacientes a unidade de saúde para recolherem o laudo do exame Papanicolaou e, com a identificação das infecções, a apresentação do exame ao profissional competente que, em casos positivos, poderá iniciar o tratamento precocemente. Todavia, esse estudo representa ainda, base de dados para fundamentar ações estratégicas que venham a contribuir para a saúde da população.

Este trabalho é uma pesquisa documental, exploratória, com abordagem quantitativa. A pesquisa documental faz uso de documentos com informações abundantes, elaborado para diversas finalidades, escritos ou não, que integram principalmente arquivos públicos (municipais, estaduais e nacionais), particulares (principalmente: domicílios particulares e 
instituições privadas) e fontes estatísticas a cargo de órgãos oficiais e particulares. A pesquisa exploratória intenciona oferecer mais informações para permitir maior conhecimento do assunto abordado. Por meio do entendimento do tema, pode-se então, elaborar hipóteses, delimitar o tema e definir objetivos (Köche 2016).

O presente estudo foi realizado na Unidade de Saúde Casa Populares/PAPS, localizada na praça Irmã Fernanda, no bairro Casas Populares, município de Cajazeiras - Paraíba, Brasil. Almejou-se a realização da presente pesquisa a partir de dados provenientes de outras UBS. No entanto, isto não foi possível por não existirem registros das variáveis necessárias para o estudo no livro do exame de Papanicolaou das Unidades. Logo, o critério de escolha da unidade foi, além do volume do número de exames, o registro dos dados necessários para a pesquisa.

A população foi composta por todos os exames de Papanicolaou realizados, porém não retirados pelas pacientes, no período de janeiro de 2010 a dezembro de 2015. A amostra foi composta por 113 resultados dos exames de Papanicolaou não retirados pelas mulheres atendidas na Unidade Básica de Saúde (UBS) Casas Populares/PAPS, e que atendeu aos critérios de inclusão. Os critérios de exclusão para a não participação do estudo foram: os dados do livro de registro dos resultados dos exames que não continham todas as informações que atendessem aos objetivos do estudo.

Os dados foram coletados dos livros de registro dos resultados dos exames de Papanicolaou da UBS, realizado no mês de setembro de 2016. Foi elaborado um instrumento de coleta a partir de uma planilha no Microsoft Excel para ambiente Windows com os elementos do estudo. Entre eles, estão a presença de ISTs, o tipo de IST encontrado, bem como as alterações presentes no exame. Em tais exames foram avaliados a frequência e a porcentagem de tais aspectos.

A pesquisa foi aprovada pelo Comitê de Ética em Pesquisa do CFP/UFCG parecer número 1.478167 e CAAE: 53864316.3.00005575, seguindo as exigências éticas da Resolução 466/12.

Os laudos dos exames de Papanicolaou não retirados por mulheres durante o período de janeiro de 2010 a dezembro de 2015 mostraram que 30 (26\%) delas tiveram resultados positivos para ISTs, sendo que um destes exames apresentou positividade para duas ISTs. Os resultados negativos para essas doenças foram encontrados em $82(73 \%)$ dos laudos, ressaltando que houve mais $1(1 \%)$ resultado de alteração celular possivelmente não neoplásica (Figura 1).

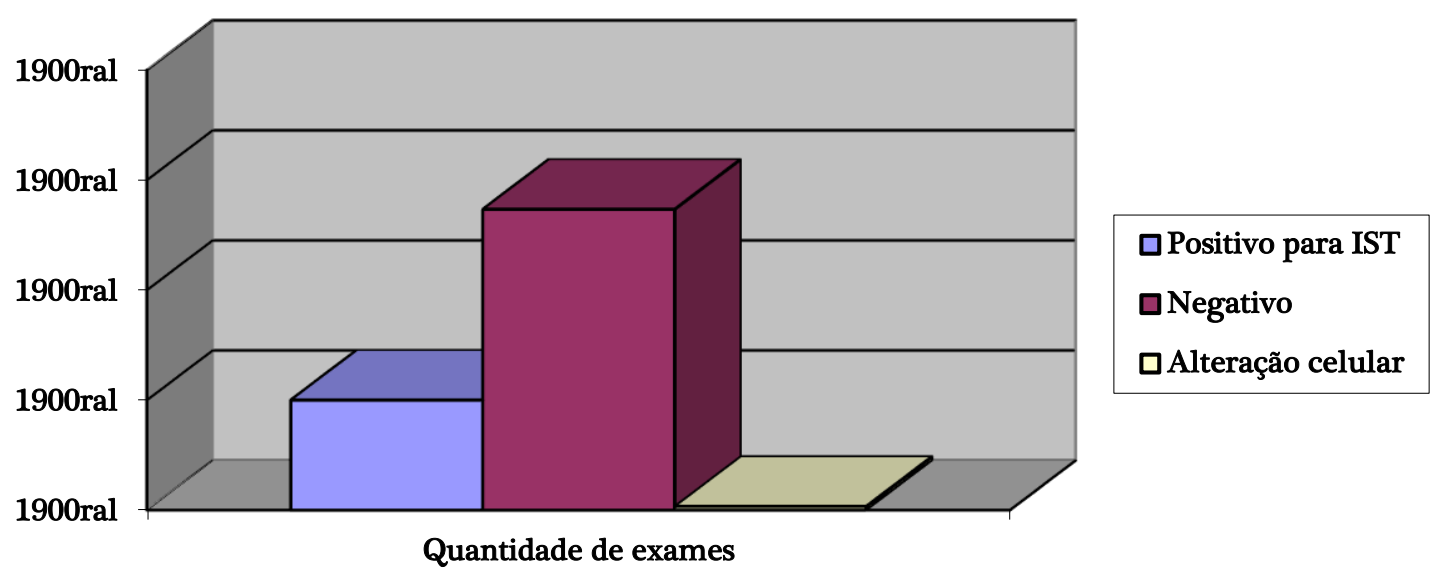

Figura 1. Resultado de exames de Papanicolaou não procurados por mulheres em uma UBS em Cajazeiras - Paraíba, entre 2010 e 2015.

Corroborando com os dados do estudo, Ferreira et al. (2018) analisaram em uma UBS no Crato - Ceará, prontuários de mulheres entre 12 e 50 anos de idade sexualmente ativas que realizaram exame citopatológico no período de 2010 a 2012 e identificaram em 31.6\% deles um ou mais microrganismo patogênico do trato geniturinário feminino, sendo eles Trichomonas vaginalis (3.3\%), Gardnerella vaginallis (19.5\%) e Candida sp. (7.6\%). Além disso, Barcelos et al. (2008) ao descrever prevalência para infecções genitais em mulheres de 15 a 49 anos atendidas 
em uma UBS em Vitória - Espírito Santo, demonstraram 3.3\% de alterações citológicas que sugerem infecção por papilomavírus humano (HPV).

Para tanto, é importante compreender os motivos do não comparecimento para receber os resultados do exame. Silva et al. (2016) investigaram os motivos do não comparecimento ao exame preventivo Papanicolaou das mulheres que realizaram o exame em 2012, mas não houve a repetição em 2013. Dos motivos apresentados por elas, o preponderante foi acreditarem não precisar realizá-lo por estarem bem de saúde. Já Lopes et al. (2015) realizaram um estudo com 11 mulheres que estavam com resultados de exame Papanicolaou na unidade de saúde realizado há mais de 60 dias e dentre as explicações relatadas pelas mulheres que não foram receber o exame, destacaram-se: demora na chegada do resultado, falta de tempo da usuária, gravidez, residência fora da área de abrangência da unidade de saúde e a não conscientização das mesmas sobre a importância do exame.

Dentre os fatores que impedem o retorno da mulher para buscar o resultado do exame, Greenwood et al. (2006) mencionaram a longa espera para o atendimento ou a marcação de uma consulta e indisponibilidade de recursos materiais e humanos do serviço. Sendo assim, provavelmente existiu a presença de algumas destas questões para a quantidade de exames que foram deixados na unidade de saúde onde foi realizada a pesquisa.

Na Tabela 1, os resultados mostram que dos 30 resultados positivos dos laudos de exame Papanicolaou referentes às ISTs, $22(71 \%)$ corresponderam aos casos de Vaginose e $9(29 \%)$ aos casos de Candidíase. Vale ressaltar que em um dos laudos foi observado a Vaginose e candidíase simultaneamente.

Tabela 1. Frequência dos tipos de ISTs encontradas nos exames positivos de mulheres que não procuraram os resultados do exame Papanicolaou em uma UBS em Cajazeiras - Paraíba entre 2010 e 2015.

\begin{tabular}{lcc}
\hline Resultados & N & $\%$ \\
\hline Vaginose bacteriana & 22 & 71 \\
Candidíase & 9 & 29 \\
\hline Total & 31 & $\mathbf{1 0 0} \%$ \\
\hline
\end{tabular}

Os resultados do presente estudo tem semelhança com outros encontrados no Brasil, como o estudo retrospectivo de Laganá et al. (2013) sobre "Alterações citopatológicas, doenças sexualmente transmissíveis e periodicidade dos exames de rastreamento em uma unidade básica de saúde" realizado na zona sul de Natal - Rio Grande do Norte, que obtiveram como achados mais frequentes laudos com resultados de Gardnerella vaginalis e Candida sp., respectivamente, sendo estes responsáveis pelo surgimento da Vaginose e candidíase.

A pesquisa produzida por Pinheiro et al. (2014), a qual realizou uma análise dos exames colpocitológicos não retirados por pacientes entre 2007 e 2009 em um Centro de Saúde da Família na periferia de Fortaleza - Ceará, identificou a Vaginose e candidíase, respectivamente, como sendo as ISTs mais presentes nos laudos.

No estudo realizado por Batista et al. (2012), "Resultados citopatológicos de mulheres que realizaram exame do colo do útero em um laboratório escola da Universidade Federal de Goiás, Goiânia-GO: estudo de prevalência”, observou-se a Vaginose, candidíase e a Trichomoniase, respectivamente, como sendo as principais infecções vaginais.

Com base nos dados do presente estudo, constatou-se que 113 mulheres deixaram de procurar os laudos do exame de Papanicolaou na UBS em Cajazeiras. Apesar da pesquisa ter sido realizada em apenas uma UBS, o número é preocupante, tendo em vista que o município de Cajazeiras tem vinte e quatro UBS, podendo haver um número ainda mais expressivo.

Diante do desperdício de tempo e recursos, por parte do serviço e da mulher, pois o objetivo do exame não é alcançado, é necessário o retorno das pacientes para recolherem o resultado do exame e a apresentação do mesmo ao profissional competente que, nos casos 
positivos, poderá iniciar o tratamento precocemente, impedindo a transmissão e evitando outras infecções e prováveis complicações.

É imprescindível que os serviços estejam atentos para o rastreamento das mulheres, utilizando ações que facilitem o acesso delas ao serviço, gerando nos profissionais um senso de responsabilidade em relação ao acolhimento das pacientes, para trazer maior efetividade ao serviço e, dessa forma, contribuir na redução do número de laudos não retirados pelas pacientes.

\section{Referências}

Barcelos M.R.B., Vargas P.R.M., Baroni C. \& Miranda A.E. (2008) Infecções genitais em mulheres atendidas em Unidade Básica de Saúde: prevalência e fatores de risco. Revista Brasileira de Ginecologia e Obstetrícia, 30(7): 349-354. https://doi.org/10.1590/S0100-72032008000700005

Batista M.L.S., Cintra A.C.F., Santos J.P.C., Martins P.D., Ribeiro A.A., Tavares S.B.N., Passos X.S. \& Alcântara K.C. (2012) Resultados citopatológicos de mulheres que realizaram exame do colo do útero em um laboratório escola da Universidade Federal de Goiás, Goiânia-GO: estudo de prevalência. Journal of the Health Sciences Institute, 30(3): 201-205.

BRASIL (2013) Ministério da Saúde. Controle dos cânceres do colo de útero e da mama. $2^{\circ}$ edição. Brasília: Ministério da Saúde.

BRASIL (2015) Ministério da Saúde. Secretaria de Ciência, Tecnologia e Insumos Estratégicos. Protocolo Clínico e Diretrizes Terapêuticas Infecções Sexualmente Transmissíveis. Brasília: Ministério da Saúde. Disponível em: https://bvsms.saude.gov.br/bvs/publicacoes/protocolo_cli nico_diretrizes_terapeutica_atencao_integral_pessoas_infeccoes_sexualmente_transmissiveis.pd f (Acesso em: 19/11/2020).

BRASIL (2016) Instituto Nacional de Câncer José Alencar Gomes da Silva. Coordenação de Prevenção e Vigilância. Divisão de Deteç̧ão Precoce e Apoio à Organização de Rede. Diretrizes brasileiras para o rastreamento do câncer do colo do útero. $2^{\circ}$ edição. Rio de Janeiro: INCA. Disponível em: https://www.inca.gov.br/publicacoes/livros/diretrizesbrasileiras-para-o-rastreamento-do-cancer-do-colo-do-utero (Acesso em: 12/10/2020).

BRASIL (2020) Ministério da Saúde. Secretaria de Atenção à Saúde. Instituto Nacional de Câncer. Coordenação de Prevenção e Vigilância de Câncer. Estimativa 2020: incidência de câncer no Brasil. Rio de Janeiro: INCA. Disponível em: https://www.inca.gov.br/publicacoes/livros/estima tiva-2020-incidencia-de-cancer-no-brasil (Acessado em: 20/11/2020).

Ferreira R.J., Vieira C.EN., Vieira M.S. \& Melanda G.C.S. (2018) Perfil epidemiológico de mulheres submetidas ao exame citopatológico em uma unidade básica. Cadernos de Cultura e Ciência, 17(1): 36-51. https://doi.org/10.14295/cad.cult.cienc.v17i1.1499

Greenwood S.A., Machado M.F.A.S. \& Sampaio N.M.V. (2006) Motivos que levam mulheres a não retornarem para receber o resultado de exame Papanicolau. Revista latino-americana de Enfermagem, 14(4): 503-509.

Köche J.C. (2016) O fluxograma da pesquisa científica (p. 121-136). In: Matos A.P.S., Silva J.M., Peretti L. \& Oleniki M.L. (Eds). Fundamentos de metodologia científica: teoria da ciência e iniciação à pesquisa. Petrópolis: Editora Vozes. 182 p.

Laganá M.T.C., Silva M.M.P., Lima L.F. \& França T.L.B. (2013) Alterações citopatológicas, doenças sexualmente transmissíveis e periodicidade dos exames de rastreamento em Unidade Básica de Saúde. Revista Brasileira de Cancerologia, 59(4): 523-530. https://doi.org/10.32635/2176-9745.RBC.2004v59n1.969

Lopes J.S.O., Dutra L.R.S., Lima N.M.M., Dodt R.C.M. \& Moura D.J.M. (2015) Papanicolaou: por que mulheres não retornam para receber resultado do exame? Revista Diálogos Acadêmicos, 3(1): 55-60.

Pinheiro M.A.C.F., Vasconcelos C.T.M., Vasconcelos Neto J.A., Cunha D.F.F. \& Pinheiro A.K.B. (2014) Análise dos exames colpocitológicos não retirados por pacientes entre 2007-2009: estudo documental. Online Brazilian Journal of Nursing, 13(1): 46-52. 
Silva L.S.R., Lessa E.C., Silva T.M., Albuquerque A.K.D.S., Ferreira M.D.R. \& Silva T.L.L. (2016) Adesão ao exame Papanicolau por mulheres jovens em unidade básica de saúde. Revista de Enfermagem UFPE on line, 10(12): 4637-4645. 